\title{
Growth of colorectal polyps: redetection and evaluation of unresected polyps for a period of
}

\section{three years}

\author{
B Hofstad, M H Vatn, S N Andersen, H S Huitfeldt, T Rognum, S Larsen, M Osnes
}

\begin{abstract}
Background, Aims, and Patients-In a prospective follow up and intervention study of colorectal polyps, leaving all polyps less than $10 \mathrm{~mm}$ in situ for three years, analysis of redetection rate, growth, and new polyp formation was carried out in 116 patients undergoing annual colonoscopy. The findings in relation to growth and new polyp formation were applied to 58 subjects who received placebo.
\end{abstract}

Results-Redetection rate varied from $\mathbf{7 5}-\mathbf{9 0} \%$ for each year, and was highest in the rectum and sigmoid colon. There was no net change in size of all polyps in the placebo group, however, polyps less than $5 \mathrm{~mm}$ showed a tendency to net growth, and polyps 5-9 $\mathrm{mm}$ a tendency to net regression in size, both for adenomas and hyperplastic polyps. This pattern was verified by computerised image analysis. Patients between 50 and 60 years showed evidence of adenoma size increase compared with the older patients, and the same was true for those with multiple adenomas (four to five) compared with those with a single adenoma. The new adenomas were significantly smaller and $71 \%$ were located in the right side of the colon. Patients with multiple adenomas had more new polyps at all the follow up examinations than patients with a single adenoma. One patient developed an invasive colorectal carcinoma, which may be evolved from a previously overlooked polyp. Two polyps, showing intramucosal carcinoma after follow up for three years, were completely removed, as judged by endoscopy and histological examination.

Conclusions-The results show that follow up of unresected colorectal polyps up to $9 \mathrm{~mm}$ is safe. The consistency of growth retardation of medium sized polyps suggests extended intervals between the encoscopic follow up examinations, but the increased number of new polyps in the proximal colon indicates total colonoscopy as the examination of choice. The growth retardation of the medium sized polyps may partly explain the discrepancy between the prevalence of polyps and the incidence of colorectal cancer.

(Gut 1996; 39: 449-456)

Keywords: colorectal polyps, redetection, growth, recurrence.
Most colorectal cancers are believed to arise in pre-existing adenomas, possibly through an accumulating succession of genetic events. ${ }^{1}$ From the small initial polyps a growth phase over many years is probable before malignant transformation may occur. ${ }^{2}$ In addition to the importance of new polyp formation, the growth rate of colorectal polyps may be of considerable interest, as the risk of malignancy in colorectal adenomas increases with size, especially in polyps more than $1 \mathrm{~cm}$ in diameter. $^{2} 3$

In a prospective intervention study of growth of colorectal polyps over three years, polyps less than $10 \mathrm{~mm}$ in maximal diameter were left in situ. ${ }^{4}$ Redetection rate, growth, and new detected polyps were evaluated at the first year follow up examination. ${ }^{5}$

The purpose of this study was to evaluate whether the macroscopic growth pattern during the first year could be verified during the second and third year. In our previous report ${ }^{5}$ we were neither aware of the histological classification of the polyps, nor did we know which intervention medication the patients used. In this study we wanted to evaluate growth and new polyp formation only in the adenomatous polyps in the patients using placebo, to avoid a possible effect of the intervention medication. Moreover, as this is the first study leaving polyps more than $5 \mathrm{~mm}$ in situ, we also needed to assess the safety aspect and the feasibility of such a study after completion.

\section{Methods}

\section{Study subjects and design}

The total study included 116 patients (male/ female 59/57) aged 50-76 years at entry. ${ }^{4}$ Polyps $\geq 10 \mathrm{~mm}$ were removed, while the rest were left in situ for a follow up period of three years with annual colonoscopic follow up examinations or removed if they reached a size beyond $9 \mathrm{~mm}$. No biopsy specimens of the polyps were taken before the end of the study. The patients received placebo or a mixture consisting of calcium and antioxidants, stratified according to the size of the polyps and block randomised, to test if the active medication was able to reduce polyp growth. ${ }^{4}$ Placebo was given to 58 patients. Included in the intervention trial (published later) was a medical and family history, which showed that non-steroidal anti-inflammatory drugs (NSAIDs) and aminosalicylic acid were used 
regularly by only two patients using placebo, and that none of the 116 patients included in the whole study had more than one first degree relative with colorectal cancer.

A measuring probe with $1 \mathrm{~mm}$ grading (polyprobe) inserted through the endoscope was applied to measure the maximal diameter of the polyps. As previously reported, ${ }^{4}$ the following variables were noted for each polyp at each endoscopic follow up examination: the size, the segmental localisation, and the distance from anus, the polyp characteristics and the relation to intraluminal structures. The base of the polyps were judged as either sessile, broad stalked or pedunculated and the body as round or elongated. Photographs with the measuring probe in position were taken for each polyp at each follow up examination. At the follow up examinations all previous data, except the diameter readings and the pictures, were available. Polyps were considered as redected when discovered at one examination and subsequently detected at the next examination.

The annual follow up examinations were scheduled to be performed within $12 \pm 1$ months after the prior examination and were carried out by one investigator. At the final follow up examination all polyps were removed. In general, polyps $5 \mathrm{~mm}$ or more in diameter were removed by snare resection $(n=96)$ and the smaller by hot biopsy $(n=404)$.

\section{Computerised image analysis}

All images of the polyps were subjected to computerised analysis, as described. ${ }^{6}$ Briefly, the images of the polyps with the measuring probe in position were visualised on a PC monitor by a videocamera. Drawing the $\mathrm{mm}$ unit and the periphery of the polyp on the screen with a light pen, the computer calculated the area of the polyp on the picture, the length of the polyp, the maximal width 90 degrees on the length, and the maximum distances from the centre of gravity. To make approximated volume analysis these variables were combined in the following way (a) polyp area $\times($ length + width $) / 2$, (b) polyp area $\times$ (maximum + minimum distance from centre of gravity to the edge of the polyp) $/ 2$, and (c) (length+width) to the third power. As a measure of the oval shape width/length was calculated.

\section{Histopathological diagnosis}

All polyps removed were evaluated by one experienced histopathologist with special interest in gastroenterological pathology, and classified according to WHO's criteria.? Dysplasia was divided into low grade and high grade according to cellular characteristics. Invasion of the lamina propria, but not through the muscularis mucosa, was termed intramucosal carcinoma. With more than $20 \%$, but less than $70 \%$ villous structures, an adenoma was termed tubulovillous. Non-neoplastic polyps included hyperplastic polyps and mucosal tags.
A sample of 65 polyps randomised within six different histological groups were selected for reproducibility testing.

\section{Statistical methods}

Except for frequencies, all results are expressed as mean values $(95 \% \mathrm{CI})$ constructed by the Student procedure. $^{8}$ All tests used in this analysis were carried out two tailed with a significance level of $5 \%$. Comparison of groups regarding frequencies were carried out by categorical data analysis. Kappa was used as a value for intraobserver agreement of histological evaluation. ${ }^{8}$

\section{Ethics}

The study was approved by the regional ethical committee. Written and oral information was given to all and oral consent required.

\section{Results}

\section{Patients and polyps at follow up}

Table I shows the patient attendance, the completeness of the colonoscopies, and colon cleansing. In the patients without a total colonoscopy at inclusion, no polyps were found proximal to the most proximal polyp at a later complete colonoscopy. Only 11 of 116 patients did not attend the final follow up examination, of whom five were dead, two had completed the study after two years, and one had moved. A total of 303 polyps were discovered in the 116 patients at inclusion, and 44 of these polyps had to be removed because of the size $(\geq 10 \mathrm{~mm}$ in diameter). Another six polyps had to be removed for the same reason at the first and second year follow up examination, two of them were follow up polyps, both receiving active medication. At the end of the study 448 polyps (256 adenomas) were removed in 104 patients (Table II). The mean number of polyps per patient at each follow up examination were $2 \cdot 6,3 \cdot 0$, and $4 \cdot 3$ respectively $(1 \cdot 4$, $1 \cdot 6$, and $2 \cdot 4$ for adenomas). Of polyps less than

TABLE I Percentage of patients attending the yearly colonoscopic follow up, with complete colonoscopies and with good bowel preparations in the total material

\begin{tabular}{lllll}
\hline & Inclusion & $\begin{array}{l}\text { First } \\
\text { year }\end{array}$ & $\begin{array}{l}\text { Second } \\
\text { year }\end{array}$ & $\begin{array}{l}\text { Third } \\
\text { year }\end{array}$ \\
\hline Patients investigated (\%) & $\mathrm{n}=116$ & 89 & 87 & 91 \\
Complete colonoscopies (\%) & 90 & 88 & 92 & 99 \\
Good colon cleansing (\%) & 77 & 65 & 75 & 79
\end{tabular}

TABLE II Number of redetected, not redetected, and newly discovered polyps at each yearly colonoscopic follow up in the total material. A total of 303 polyps were discovered at inclusion in 116 patients. Forty four polyps $\geq 10 \mathrm{~mm}$ in diameter were removed

\begin{tabular}{llcl}
\hline Polyps & \multicolumn{3}{l}{ Endoscopic follow up examination } \\
\cline { 2 - 4 } & First year & Second year & Third year \\
\hline Redetected & 176 & 222 & 330 \\
Not redetected & 48 & 75 & 55 \\
New & 84 & 83 & 118 \\
Total & 308 & 380 & 503 \\
\hline
\end{tabular}




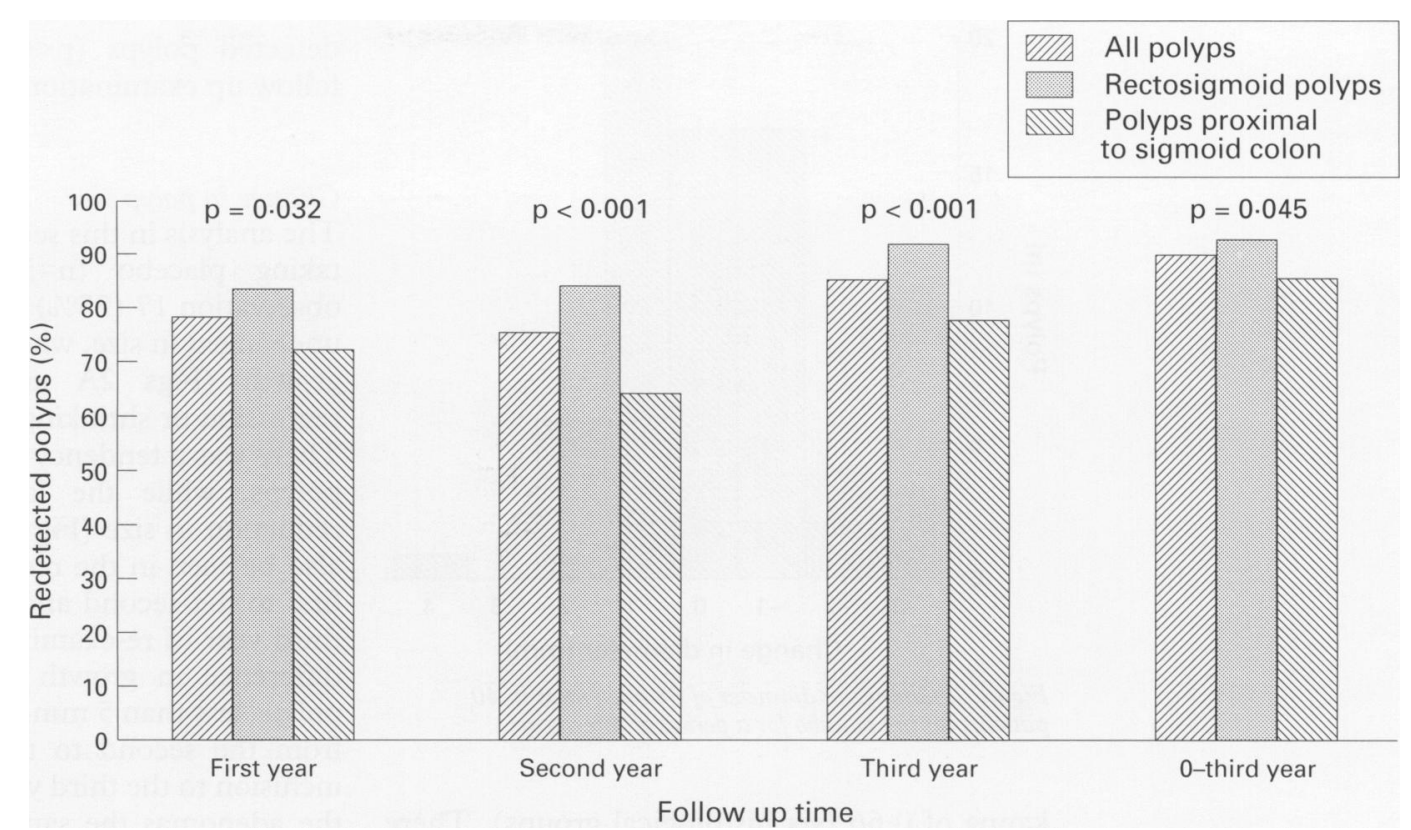

Figure 1: The redetection of all polyps (\%) at each control for all polyps and for the polyps proximal and distal to the sigmoid/descendic junction. The $\chi^{2} p$ values between redetection for the distal and proximal polyps are indicated at the top of the columns.

$5 \mathrm{~mm}$ approximately $90 \%$ were considered sessile and $75 \%$ round. About $50 \%$ of the polyps 5-9 $\mathrm{mm}$ were sessile, and $45 \%$ were round. In the right side of the colon compared with the left side significantly more polyps 5-9 $\mathrm{mm}$ in diameter were sessile $(\mathrm{p}<0.001)$ and elongated $(p<0.05)$. At inclusion, seven of 58 patients receiving placebo had only hyperplastic polyps, 29 patients had one adenoma, 17 patients had two to three adenomas, and five patients had four to five adenomas. There
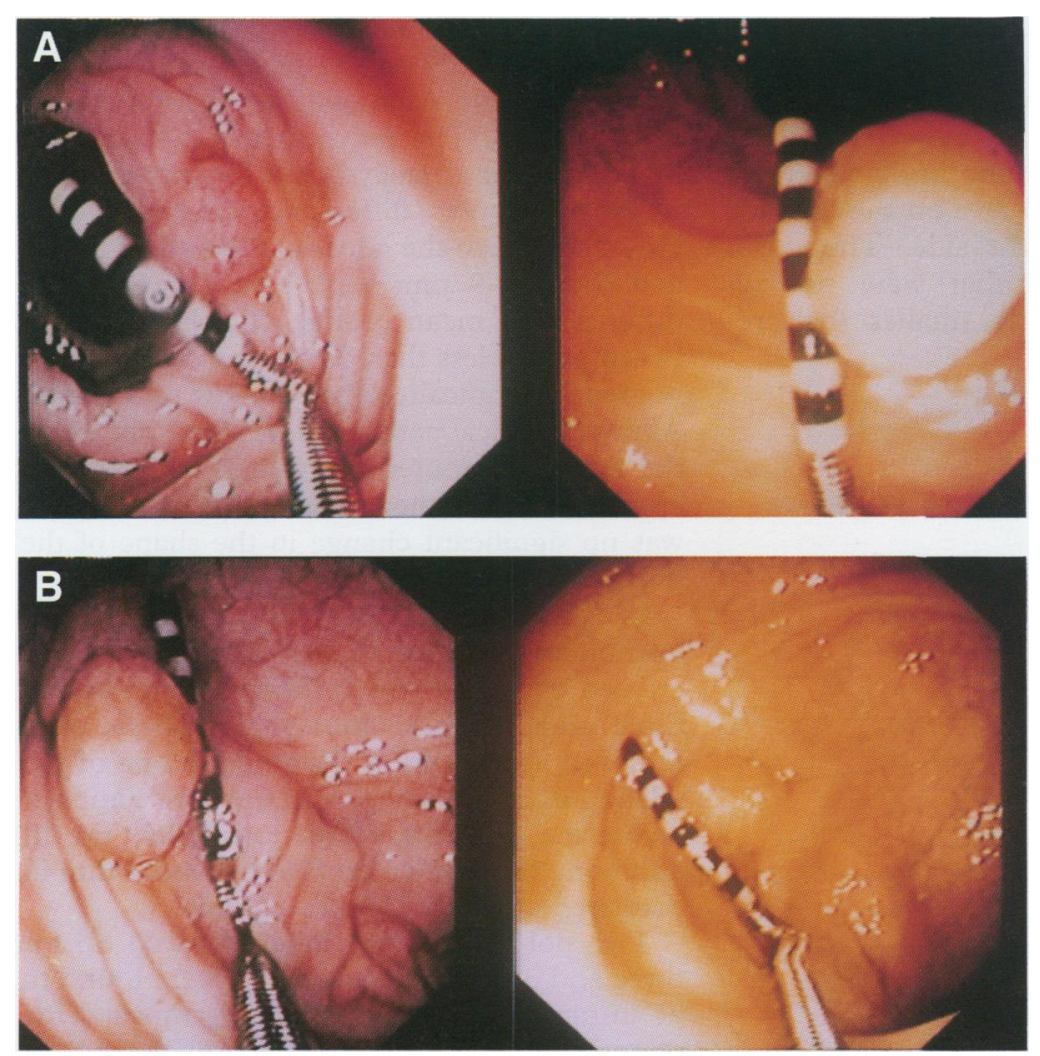

Figure 2: Photographs of two polyps followed up, as examples of increasing $(A)$ and regressing (B) polyps: $(A)$ shows a polyp increasing $2 \mathrm{~mm}$ in diameter in three years, and (B) a polyp regressing $2 \mathrm{~mm}$ in diameter in three years. A measuring probe is placed alongside the polyp for assessment of diameter. Each white and black grading is $1 \mathrm{~mm}$. was no relation between age or family history of colorectal cancer and multiplicity of adenomas.

One patient developed an invasive colon cancer during the three years of follow up. In this patient, a polyp in the ascending colon was removed at inclusion, however, a sample for histological examination was not obtained. No polyp or neoplastic remnants were found in the right colon at the first year examination. An asymptomatic $3 \mathrm{~cm}$ large invasive adenocarcinoma was detected in the ascending colon at the second year examination (Dukes's B). The patient did well after the operation, but died suddenly a year later after an episode of chest pain, before the final examination. A necropsy was not performed. Another eight patients developed cancer during follow up, two of them with liver metastasis from a colonic cancer resected prior to inclusion in the study and no signs of local recurrence. Six patients developed extracolonic cancer, of whom three died from their malignancy in the study period.

\section{Histopathological diagnosis}

Of the 494 polyps in the total material removed and evaluated histologically during the study, $62 \%$ were adenomas, and $30 \%$ hyperplastic polyps. At inclusion, adenomas were found in $51 \%$ of polyps less than $5 \mathrm{~mm}$, in $81 \%$ of polyps 5-9 $\mathrm{mm}$, and in all polyps more than $9 \mathrm{~mm}$. A total of 80 patients $(76 \%)$ had at least one polyp followed up, that proved to be an adenoma on removal. Only two polyps that were followed up displayed intramucosal carcinomas at resection. High grade dysplasia were found in four polys and tubulovillous pattern in two polyps after follow up. The rest of the adenomas showed a regular tubular pattern with low grade dysplasia $(n=194)$.

The tests on reproducibility showed a $73 \%$ complete intraobserver agreement with a 


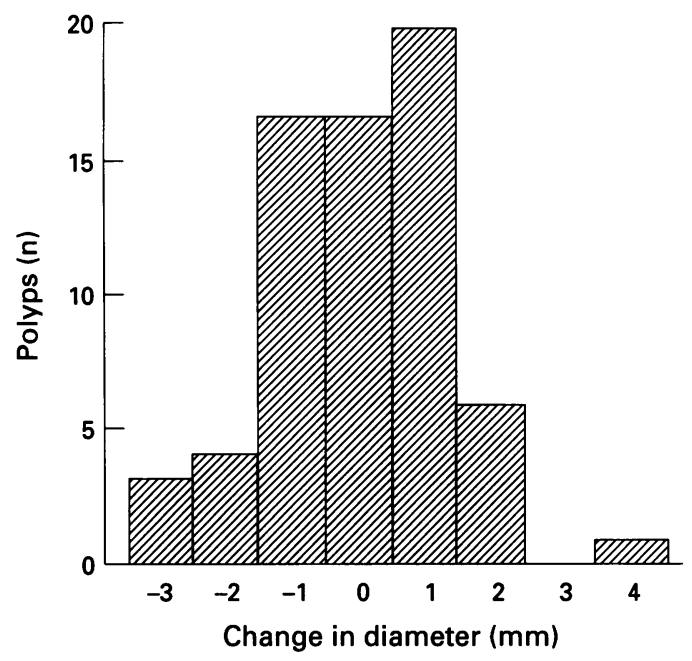

Figure 3: Change in diameter of 68 adenomas in 40 patients taking placebo for a period of three years.

kappa of 0.66 (six histological groups). There was no disagreement in the separation of neoplastic and non-neoplastic polys, but some differences in the judgement of degree of dysplasia.

\section{Redeteced polyps}

A high percentage of the polyps were redetected at each follow up examination (Fig 1), with a reduction rate ranging from 75 to $90 \%$. Table II shows the multiplicity of polyps at each examination. The redetection was significantly better in the rectum and sigmoid colon, than in the more proximal segments at all the follow up examinations $(p<0.05)$ (Fig 1$)$. This was true both for polyps less than $5 \mathrm{~mm}$ and 5-9 $\mathrm{mm}$ in diameter. The percentage of polyps less than $5 \mathrm{~mm}$ in diameter not redetected ranged from 11 to $24 \%$ in the left side of the colon to $14-46 \%$ in the right side. The percentage of polyps $5-9 \mathrm{~mm}$ in diameter not redetected ranged from $4-9 \%$ on the left side and $10-24 \%$ on the right side. The mean diameter of the polyps that were not redetected, was significantly smaller than re-

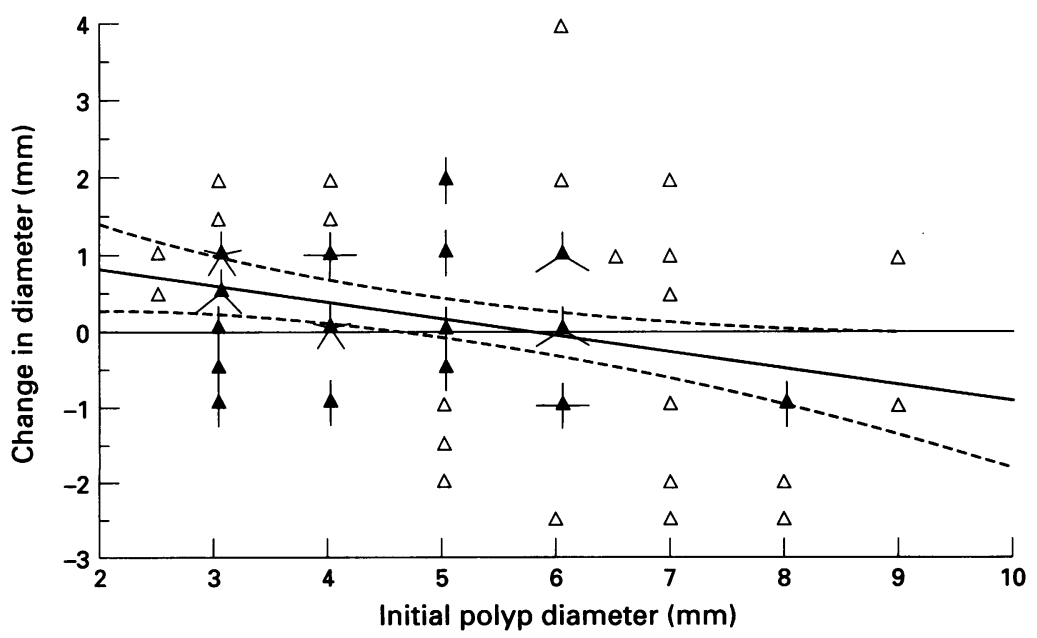

Figure 4: Change in diameter of 68 adenomas in 40 patients taking placebo after an observation period of three years, in relation to the initial size of the polys. The scatterplot is presented as flower diagram in which each line emanating from a point represents an exact overlap. Non-overlapping registrations are presented as triangles. Regression line with confidence limits are drawn. detected polyps $(\mathrm{p}<0.01)$ at all the annual follow up examinations.

\section{Change in polyp size}

The analysis in this section is based on patients taking placebo $(n=58)$. After three years observation $17(25 \%)$ of all the adenomas were unchanged in size, whereas $27(40 \%)$ displayed growth (Figs 2A and 3) and 24 (35\%) regression or shrinking in size (Figs $2 \mathrm{~B}$ and 3 ). There was a tendency for growth in the smaller polyps, while the larger polyps showed a reduction in size (Fig 4). This tendency could also be seen in the redetected polyps from the first to the second and from the second to the third year of re-examination, with a significant difference in growth between the groups of polyps less than $5 \mathrm{~mm}$ and $5-9 \mathrm{~mm}$ in diameter from the second to the third year and from inclusion to the third year of re-examination. In the adenomas the same pattern was observed for all the endoscopic follow up examinations (Fig 5), also reaching statistical significance from the second to the third year. Moreover, the hyperplastic polyps showed a similar pattern, significantly different from the first year to second year of re-examination $(p \leq 0.05)$ (Fig 6). There was a tendency to increased growth in the adenomatous polyps in the younger age groups reaching significance from inclusion to the third year and from the first to the second year of re-examination (Fig 7). Moreover, in the patients with four to five adenomas at inclusion the polyps showed larger growth than the polyps in the patients with only one or two to three adenomas (Fig 8). There were no differences in polyp growth between the sexes $(p=0.24-0.87)$.

A total of $88 \%$ of the polyps were photographed at inclusion and at the follow up examinations. The polyp area was calculated by computerised analysis. As for the diameter readings, similar differences in mean area growth between the adenomas less than $5 \mathrm{~mm}$ and those 5-9 $\mathrm{mm}$ were seen (Fig 9). The difference in mean change of polyp area between polyps less than $5 \mathrm{~mm}$ and 5-9 $\mathrm{mm}$ did not reach significance at any follow up interval. Almost identical patterns were seen combining the other computerised values to make an approximate volume or mass estimation. There was no significant change in the shape of the polyps.

In the total study nine polyps (eight adenomas and one hyperplastic polyp) increased beyond $9 \mathrm{~mm}$ in diameter after one to three years of observation, of which six were removed at the end of the study. None of the adenomas in the placebo group were removed before the end of the three year follow up. In addition, 24 polyps increased beyond $4 \mathrm{~mm}$ in diameter, of which 13 were adenomas.

Two polyps that had been followed up for three and one year, respectively, were found to be intramucosal carcinomas on removal. One regressed from 9 to $7 \mathrm{~mm}$ in diameter over three years, while the other increased in size from 8 to $11 \mathrm{~mm}$ in diameter in one year before removal. Another four tubular adenomas with 


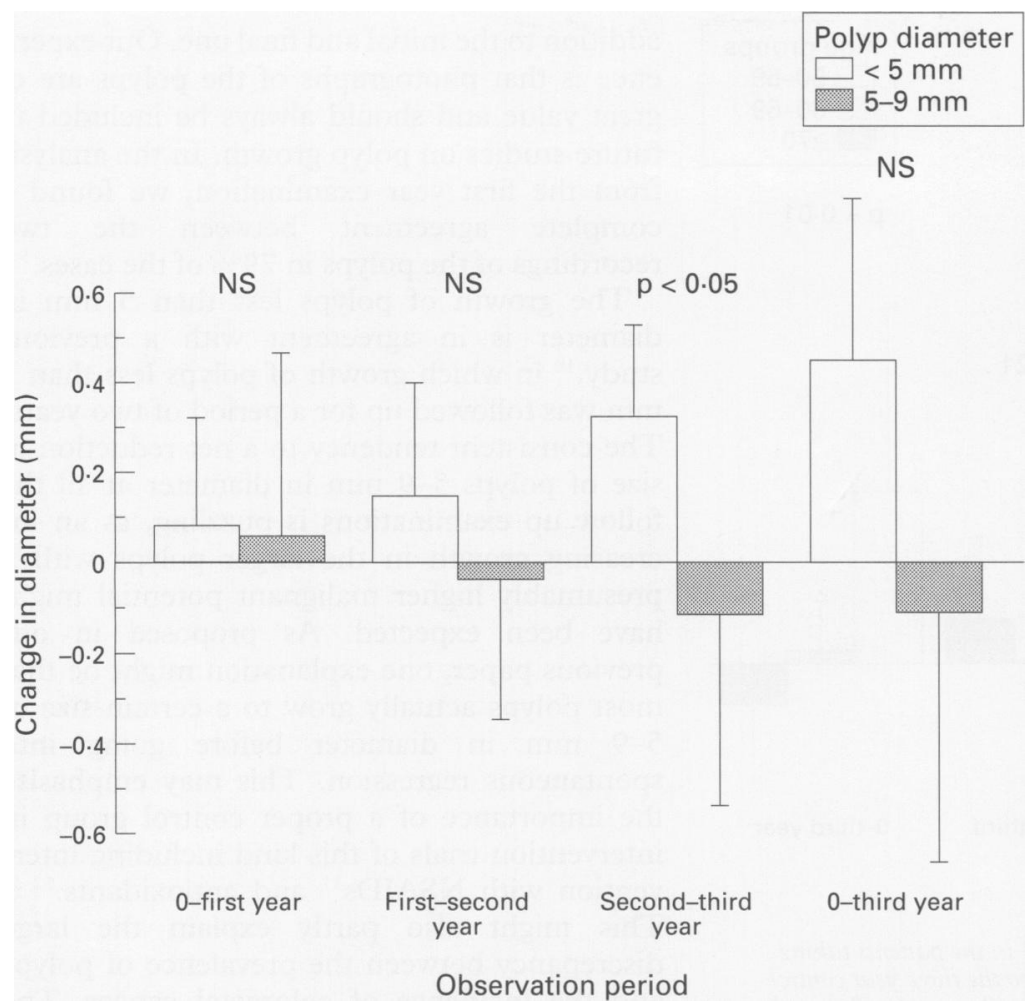

Figure 5: Mean change in diameter of the adenomas (95\% CI) in the patients taking placebo at each yearly endoscopic follow up and from inclusion to the three year control ( $0-3$ years) for the polyps $<5 \mathrm{~mm}(n=27-43)$ and the polyps $5-9 \mathrm{~mm}$ in diameter $(n=23-43)$. The $p$ values for the differences between growth of the smaller and larger polyps are shown.

high grade dysplasia were followed up for two to three years. One grew from $8 \mathrm{~mm}$ to $12 \mathrm{~mm}$ in two years before removal. The remaining three polyps showed minimal changes $( \pm 1$ $\mathrm{mm}$ ) over three years.

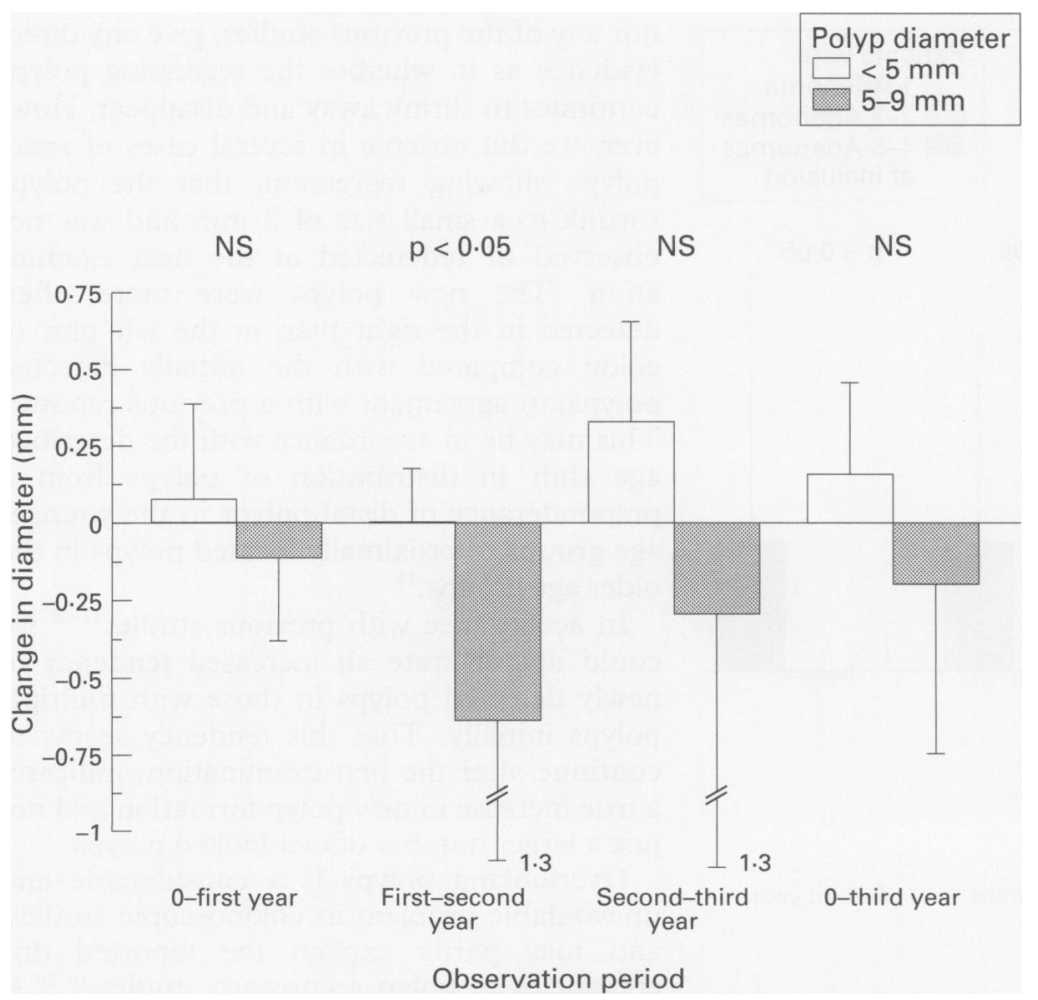

Figure 6: Mean change in diameter of the hyperplastic polyps $(95 \% \mathrm{CI})$ in the patients taking placebo at each yearly endoscopic follow up and from inclusion to the three year control ( $0-3$ years) for the polyps $<5 \mathrm{~mm}(n=7-23)$ and the polyps 5-9 $\mathrm{mm}$ in diameter $(n=5-7)$. The $p$ values for the differences between growth of the smaller and larger polyps are indicated on top.
New polyps

All the analysis in this section is also based on patients taking placebo $(n=58)$. From $19 \%$ to $31 \%$ of all the adenomas discovered at each annual follow up examination were considered as new. Of the 258 polyps removed, 23 at inclusion and 235 at the end of the study, 144 $(56 \%)$ had been discovered at the follow up examinations after inclusion. A total of $86 \%$ of the patients had at least one new polyp during the three years and $75 \%$ had at least one new adenoma. The newly discovered polyps were significantly smaller than the average size at inclusion $(p<0.001)$. They were also more frequent in the proximal part of the colon $(71 \%)$ than the polyps discovered at inclusion $(38 \%)(\mathrm{p}<0.05)$. For all the annual follow up examinations and from inclusion to the third year of examination, there were more new adenomas among those with more than four to five adenomas at inclusion $(p<0.001)$, than those with one adenoma, reaching significance from inclusion to the first year of examination, and from inclusion to the third year (Fig 10). There were more new adenomas among patients $\geq 60$ years of age than those $<60$ years $(p=0 \cdot 05)$. No differences were found between the sexes.

\section{Discussion}

The follow up of polyps in situ, including repeated evaluation of size, is connected with several methodological problems. We have previously shown that this method is reliable ${ }^{6}$ and that the measurement readings are similar using either fibreoptic endoscope or videoendoscope. ${ }^{9}$ Because the whole study relies entirely on these measurements, we have added the two dimensional area measurements of the polyp by computerised picture analysis to confirm the results.

In a previous study, measuring only polyps less than $5 \mathrm{~mm}$, the authors suggested that most polyps of this size assumed the shape of a half sphere, and applied a formula for this to calculate the change in polyp mass. ${ }^{10}$ This amplifies the growth changes to the third degree. Hence, a mean of $16 \%(0.5 \mathrm{~mm})$ increase in diameter from $3.1 \mathrm{~mm}$, resulted in an increased mass of $53 \%$. However, as this suggested shape may be true for most of the smaller polyps, no apparent unified shape can be applied for the larger polyps $5-9 \mathrm{~mm}$ in diameter. An approximate calculation of the mass for many of the oval or irregular polyps in this size range would require at least two measurements for each polyp. We have several polyp measurements applying computerised image analysis, but this method correlates less well to the mass or volume of the polyp, ${ }^{6}$ possibly owing to technical problems in getting the polyp and measuring probe in correct position. Also in this study the changes in mass would be expected to be even more pronounced than the linear differences presented.

Whenever there were multiple polyps within one colonic segment, a major problem was to decide which polyps were redetected and 


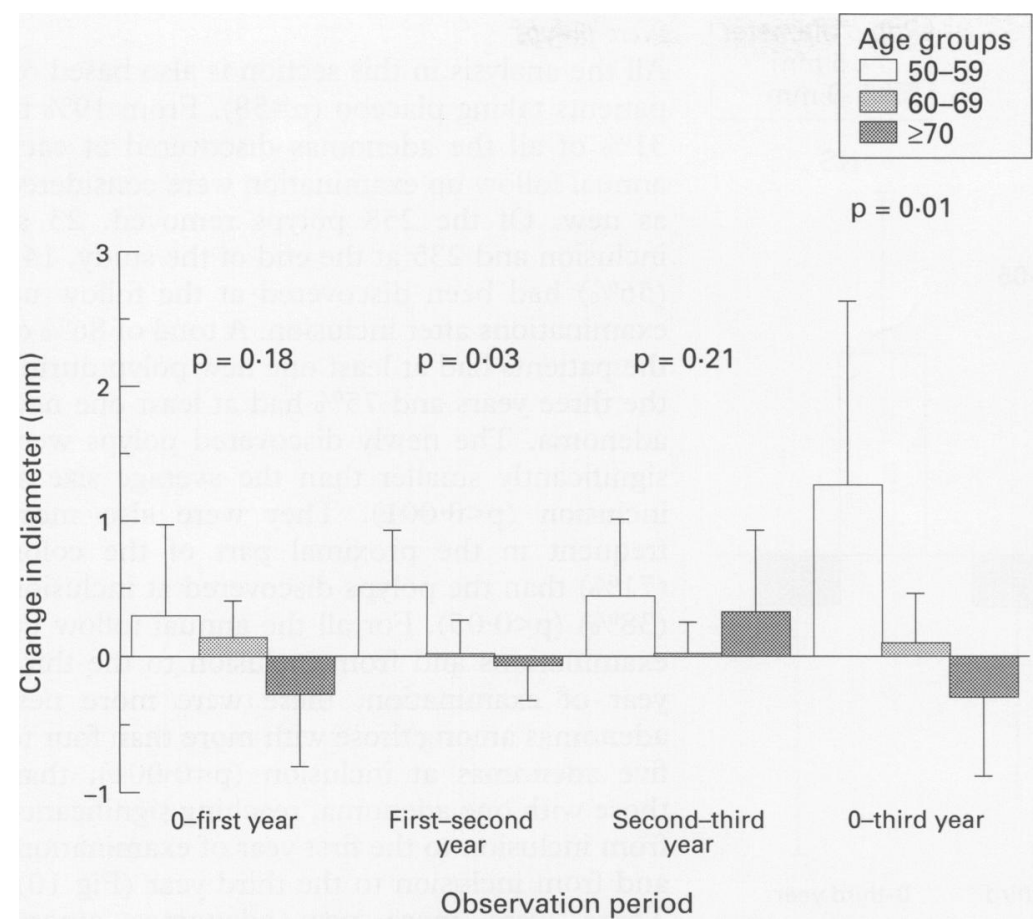

Figure 7: Mean change in diameter of the adenomas (95\% CI) in the patients taking placebo at each yearly endoscopic follow up and from inclusion to the three year control ( $0-3$ years) for patients in age groups $50-59$ years $(n=10), 60-69$ years $(n=34)$ and $\geq 70$ years $(n=14)$. The $p$ values for the differences between growth of the polyps in the youngest and oldest age groups are indicated on top.

which were new discoveries. We had several data available for each polyp (location, configuration, and size measurements) at each endoscopic follow up examination, including a picture, to aid in the final decision at the end of the study. In this respect, it was beneficial to have the two intermediate recordings, in

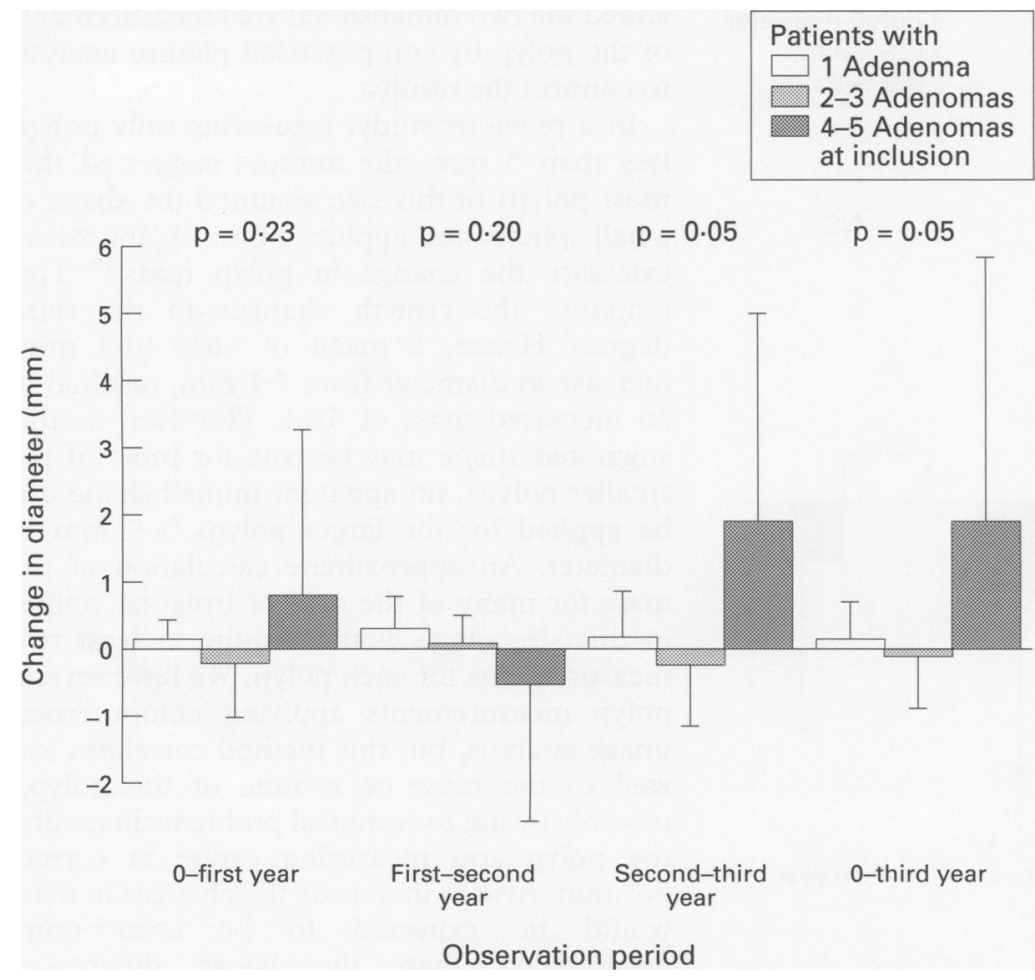

Figure 8: Mean change in diameter of the adenomas (95\% CI) in the patients taking placebo at each yearly endoscopic follow up and from inclusion to the three year control (0-3 years) for patients with one adenoma $(n=29)$, two to three adenomas $(n=17)$, and four to five adenomas $(n=5)$ at inclusion. The $p$ values for the differences between those with one initial adenoma and those with four to five are shown. addition to the initial and final one. Our experience is that photographs of the polyps are of great value and should always be included in future studies on polyp growth. In the analysis from the first year examination, we found a complete agreement between the two recordings of the polyps in $79 \%$ of the cases. ${ }^{5}$

The growth of polyps less than $5 \mathrm{~mm}$ in diameter is in agreement with a previous study, ${ }^{10}$ in which growth of polyps less than 5 $\mathrm{mm}$ was followed up for a period of two years. The consistent tendency to a net reduction in size of polyps 5-9 $\mathrm{mm}$ in diameter at all the follow up examinations is puzzling, as an increasing growth in the larger polyps with a presumably higher malignant potential might have been expected. As proposed in our previous paper, one explanation might be that most polyps actually grow to a certain size of 5-9 $\mathrm{mm}$ in diameter before going into spontaneous regression. This may emphasise the importance of a proper control group in intervention trials of this kind including intervention with NSAIDs ${ }^{11}$ and antioxidants. ${ }^{12} 13$ This might also partly explain the large discrepancy between the prevalence of polyps and the incidence of colorectal cancer. The determination of the final growth pattern of polyps, however, requires a much longer observation period than three years. Nevertheless, the moderate tendency to net regression of moderate sized polyps in this study was a constant finding for all the three years. That both adenomas and hyperplastic polyps regressed, as has been seen in two previous studies, ${ }^{10}{ }^{14}$ may lead to speculations of circulatory insufficiency or that important promotors are necessary for further growth in addition to the initiating factors. Neither this, nor any of the previous studies, give any direct evidence as to whether the regressing polyps continues to shrink away and disappear. However, we did observe in several cases of small polyps showing regression, that the polyps shrunk to a small size of $2 \mathrm{~mm}$ and was not observed or redetected at the next examination. The new polyps were more often detected in the right than in the left part of colon compared with the initially detected polyps, in agreement with a previous report. ${ }^{15}$ This may be in accordance with the described age shift in distribution of polyps from a preponderance of distal polyps in the younger age groups to proximally located polyps in the older age groups. ${ }^{16}$

In accordance with previous studies ${ }^{17-26}$ we could demonstrate an increased tendency to newly detected polyps in those with multiple polyps initially. That this tendency seems to continue after the first examination, indicates a true increase in new polyp formation and not just a larger number of overlooked polyps.

Overlooking polyps is a considerable and unavoidable problem in colonoscopic studies, and may partly explain the reported discrepancies in polyp recurrence studies. ${ }^{18} 20 \mathrm{~A}$ miss rate of $16-27 \%$ of polyps $\leq 5 \mathrm{~mm}$ in diameter and $5-17 \%$ of polyps $6-9 \mathrm{~mm}$ in diameter has been reported in prospective repeat colonoscopy studies both by the same 


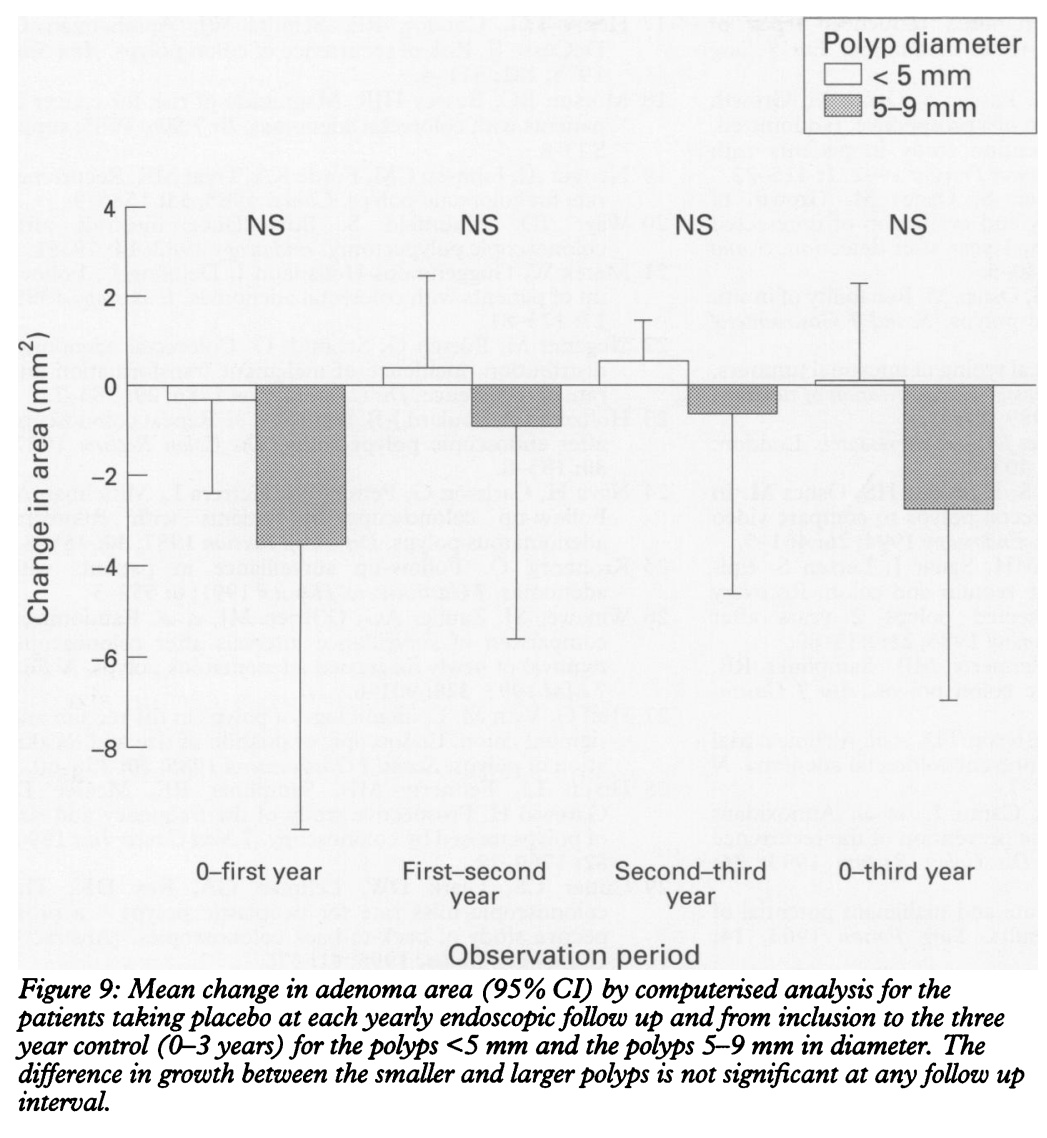
interval.

and different endoscopists. ${ }^{27-29}$ A repeat colonoscopy within a short time, would reduce the number of overlooked polyps at inclusion in our study, but was considered a too heavy burden on the patients, who were scheduled for annual endoscopic follow up colonoscopies, diet registration, and intervention medication.

For the histopathological diagnosis, there was a complete intraobserver agreement with regard to neoplasic and non-neoplastic polyps. The variation in the judgement of degree of dysplasia in adenomas has also been shown in

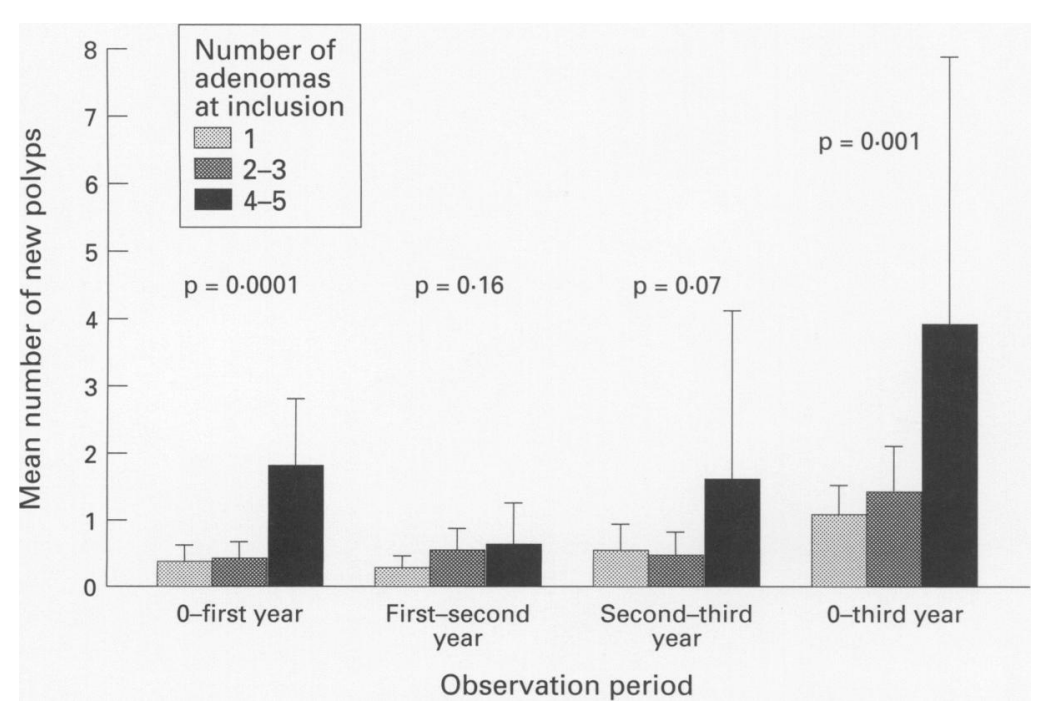

Figure 10: Number of new adenomas at each endoscopic follow up examination for patients with one $(n=29)$, two to three $(n=17)$, and four to five adenomas $(n=7)$ at inclusion. Only patients taking placebo are included. Patients with no adenomas $(n=7)$ only had two polyps at follow up and are not included in the Figure. Mean values with $95 \%$ CI are given. The $p$ values comparing those with one initial adenoma and those with four to five are shown above the columns. a previous study both for the same and different pathologists, ${ }^{30}$ indicating that a large part of the evaluation is based on opinions. In our study there were too few polyps with high grade dysplasia or tubulovillous structure, to permit statistical analysis on these subgroups. Hence, the discrepancies are of no importance for our results of growth and redetection rate.

We did not perform biopsies of the polyps prior to the final resection. A biopsy of polyps may change the growth pattern, affect the dysplasia in adenomas, and reduce the mass considerably. Moreover, high grade dysplasia or invasive malignancy, which could be the most important findings to detect, is unevenly distributed and might not have been included in a random biopsy.

The safety aspect of not removing polyps up to $9 \mathrm{~mm}$ in diameter in this study needs to be evaluated. As practically no polyps less than 5 $\mathrm{mm}$ are malignant, ${ }^{3}$ invasive cancer may be found in $0.5-0.9 \%$ of polyps from 5 to $10 \mathrm{~mm}$ in diameter. ${ }^{3}{ }^{31}$ Two polyps that were followed up in our study for one and three years, respectively, were diagnosed as intramucosal carcinomas on removal. In addition, four polyps that were left for follow up, showed high grade dysplasia after polypectomy. All six polyps were radically removed, judged by histology and endoscopy, with no signs of local recurrence at later follow up. One patient developed an asymptomatic invasive carcinoma (Dukes's B) at the second year examination, but this was not caused by a polyp being left behind. It might have evolved from a polyp that was removed in the same region at inclusion, but lost to histological examination.

In conclusion, our three years of follow up study of colorectal polyps has shown that leaving polyps up to $1 \mathrm{~cm}$ in diameter in situ for research, may be considered safe, in terms of avoiding development of invasive carcinomas, provided that annual endoscopic follow up examinations are carried out. We have confirmed our experience from the first year examination ${ }^{5}$ that the redetection of polyps left unresected was high, especially in the rectum and sigmoid colon. We have also confirmed that polyps less than $5 \mathrm{~mm}$ showed a tendency to growth, while the polyps $5-9 \mathrm{~mm}$ showed a tendency to reduction in size, a difference seen both for adenomas and hyperplastic polyps. The regression of medium sized polyps may lead to a less aggressive attitude toward colorectal polys. However, the fact that the new polyps appear more often in the proximal colon, does not lend support to the view that sigmoidoscopy alone is a satisfactory method for follow up of these patients. The regression of the medium sized polyps may partly explain the discrepancy between the prevalence of polyps and the incidence of colorectal cancer.

This study was supported by Hydropharma $\mathrm{a} / \mathrm{s}$ and The Norwegian Cancer Society.

1 Vogelstein B, Fearon ER, Hamilton SR, et al. Genetic alterations during colorectal-tumor development. $N$ Engl f Med 1988; 319: 525-32.

2 Morson BC. The polyp-cancer sequence in the large bowel. Proc Roy Soc Med 1974; 67: 451-7. 
3 Hermanek P. Dysplasia-carcinoma sequence, types of adenomas and early colo-rectal carcinoma. Eur 7 Surg Oncol 1987; 13: 141-3.

4 Hofstad B, Vatn M, Hoff G, Larsen S, Osnes M. Growth of colorectal polpys. Design of a prospective, randomized, placebo controlled intervention study in patients with placebo controlled intervention study in patients with

5 colorectal polyps. Eur f Cancer Prevent 1992; 1: 415-22. colorectal polyps: recovery and evaluation of unresected colorectal polyps: recovery and evaluation of unresected polyps of less than $10 \mathrm{~mm}, 1$ year

6 Hofstad B, Vatn M, Larsen S, Osnes M. Reliability of in situ meaurements of colorectal polyps. Scand $\mathcal{F}$ Gastroentero 1992; 27: 59-64

7 Jass JR, Sobin LH. Histological typing of intestinal tumours. In: WHO International histological classification of tumours. Berlin: Springer Verlag, 1989: 29-31.

8 Altman DG. Practical statistics for medical research. London: Chapman and Hall, 1991: 403-8.

9 Hofstad B, Vatn M, Larsen S, Huitfeldt HS, Osnes M. In situ measurement of colorectal polyps to compare video situ measurement of colorectal polyps to compare video

10 Hoff G, Foerster A, Vatn MH, Sauar J, Larsen S. Epidemiology of polyps in the rectum and colon. Recovery and evaluation of unresected polyps 2 years

1 Hixson LJ, Earnest DL, Fennerty MB, Sampliner RE. NSAID effect on sporadic colon polyps. $A m \mathcal{f}$ Gastroenterol 1993; 88: 1652-6.

12 Greenberg ER, Baron JA, Tosteson TD, et al. A clinical trial of antioxidant vitamins to prevent colorectal adenoma. $N$ Engl f Med 1994; 331: 141-7.

13 Roncucci L, Di Donato P, Carati L, et al. Antioxidant vitamins or lactulose for the prevention of the recurrence of colorectal adenomas. Dis Colon Rectum 1993; 36: $227-34$

14 Knoernschild HE. Growth rate and malignant potential of colonic polyps: Early results. Surg Forum 1963; 14: $137-8$

15 Woolfson IK, Eckholdt GJ, Wetzel CR, et al. Usefulness of performing colonoscopy one year after endoscopic polypectomy. Dis Colon Rectum 1990; 33: 389-93.

16 Vatn MH, Myren J, Serck-Hanssen A. The distribution of polyps in the large intestine. Ann Gastroenterol Hepatol 1985; 21: 239-45.
17 Henry LG, Condon RE, Schulte WJ, Aprahamian C, DeCosse JJ. Risk of recurrence of colon polyps. Ann Surg 1975; 182: 511-4.

18 Morson BC, Bussey HJR. Magnitude of risk for cancer in patients with colorectal adenomas. Br f Surg 1985; suppl:

19 Neugut AI, Johnsen CM, Forde KA, Treat MR. Recurrence rate for colorectal polyps. Cancer 1985; 55: 1586-9.

20 Waye JD, Braunfeld S. Surveillance intervals after colonoscopic polypectomy. Endoscopy 1982;14: 79-81.

21 Matek W, Guggenmoos-Holzmann I, Demling L. FollowMatek W, Guggenmoos-Holzmann I, Demling L. Follow-
up of patients with colorectal adenomas. Endoscopy 1985; 17: $175-81$.

22 Wegener M, Börsch G, Schmidt G. Colorectal adenomas: distribution, incidence of malignant transformation and rate of recurrence. Dis Colon Rectum 1986; 29: 383-7.

23 Holtzman R, Poulard J-B, Bank S, et al. Repeat colonoscopy after endoscopic polypectomy. Dis Colon Rectum 1987; 30: $185-8$.

24 Nava H, Carlsson G, Petrelli NJ, Herrera L, Mittelman A. Follow-up colonoscopy in patients with colorectal adenomatous polyps. Dis Colon Rectum 1987; 30: 465-8.

25 Kronborg O. Follow-up surveillance in patients with adenomas. f Gastroenterol Hepatol 1991; 6: 552-3.

26 Winawer SJ, Zauber AG, O'Brien MJ, et al. Randomized comparison of surveillance intervals after colonoscopic comparison of surveillance intervals after colonoscopic removal of newly diagnosed

27 Hoff G, Vatn M. Epidemiology of polyps in the rectum and igmoid colon. Endoscopic evaluation of size and localization of polyps. Scand 7 Gastroenterol 1985; 20: 356-60

28 Hixon LJ, Fennerty MB, Sampliner RE, McGee D, Garewal $H$. Prospective study of the frequency and size of polyps missed by colonoscopy. $\mathcal{F}$ Natl Cancer Inst 1990; 82: 1769-72.

29 Cutler CS, Clark DW, Lehman GA, Rex DK. The colonoscopic miss rate for neoplastic polyps - a prospective study of back-to-back colonoscopies. [Abstract]. pective study of back-to-back col

30 Brown LJR, Smeeton NC, Dixon MF. Assessment of dysplasia in colorectal adenomas: an observer variation and morphometric study. F Clin Pathol 1985; 38: 174-9.

31 Shinya $\mathrm{H}$, Wolff WI. Morphology, anatomic distribution and cancer potential of colonic polyps. Ann Surg 1979; 190: 679-83. 